\title{
NOTES ON OPTICAL INSTRUMENTS AND DEVICES
}

BY

\section{J. FOSTER}

LEEDS

\section{OPHTHALMIC NEEDLE HOLDER}

The Needle Holder illustrated is a combination of the handle and scissors shank of Gillies' needle holder, and of the jaws of Quarry Silcock's ophthalmic needle holder. It is extremely useful,

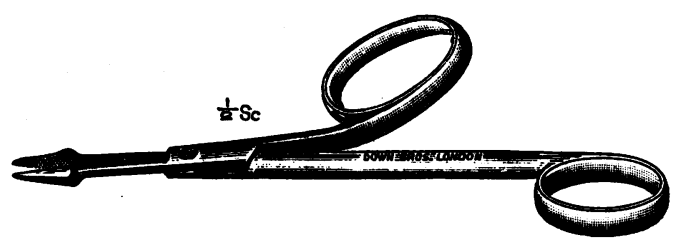

and adds to the speed of ophthalmic suturing in all situations, if the " forceps' tie " method is employed : this is specially marked in lid operations. The scissors portion is most useful except for dividing sutures near the inner canthus where there is not enough room to employ it. The instrument is made by Down Bros.

\section{THE ARTIFICIAL GLAUCOMA HALO}

OCCASIONALly one suspects a patient of having had an attack of sub-acute glaucoma. It is, however, difficult to explain to some of them exactly what a glaucoma halo looks like. A great deal of prolonged and futile description is saved if the disc (which consists of a layer of lycopodium powder between two thin sheets of glass affixed in a trial lens rim) is placed in a trial frame. Attention is directed to a Maddox spot light, and an extremely good reproduction of a glaucoma halo thus produced. A definite "yes or no" can be elicited at once.

Occasionally one encounters patients who claim that the reproduction is more, and others who claim that it is less vivid than their own halo. Very occasionally patients can see no colour in the artificial halo at all. In practice, however, I find the agreement is very close. This phenomenon was first discovered by

NotE.-These have been shown at R.S.M. meetings and some have been published in opticians' advertisements. 
Fraunhofer, and its clinical application suggested by Elliot. This device reduces the test to a convenient form for clinical use. No illustration is appended as the device is indistinguishable from an ordinary trial-case lens.

\section{TRIAL FRAME LEVEL}

Certain patients are unusually sensitive to small changes of cylinder axis position. This may be due either to the strength of the cylinder required or extremely good perceptive judgment. One also encounters patients in whom there is a considerable amount of vertical asymmetry of the face and eyes, with resultant tilting of the trial frame. A small curved bubble level filled with

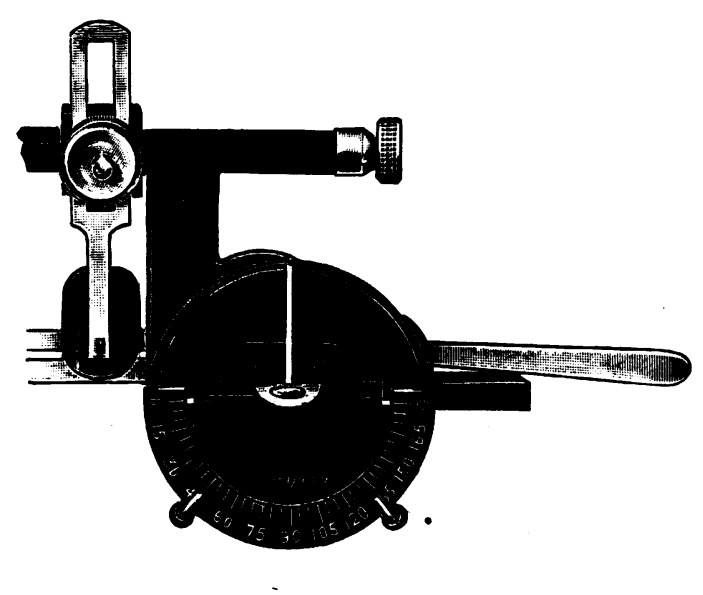

glycerine to reduce the rapidity of the bubble excursion is attached to a metal disc of the same size as an astigmatic trial lens. The horizontal line on the disc is arranged to coincide with the horizontal marks of the frame, and a constant or momentary deviation of the bubble from the centre of the level will indicate an error which must be corrected either by tilting the head or adjusting the cells or sides of the frame.

\section{THE THREE-LENS TEST}

As the effective diameter of the modern trial lens is $20 \mathrm{mms}$., it is unnecessary to employ lenses of a larger size in an end-point test; it is possible, therefore, to combine a concave sphere, a 


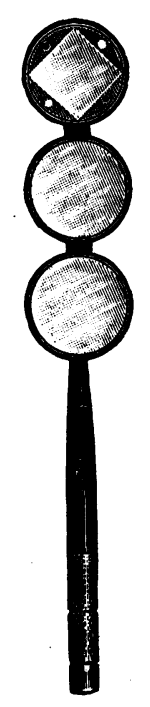

similar convex sphere, and a cross cylinder in an extremely small space and thereby to accelerate ease and speed of handling.

\section{RETINOSCOPY RACK}

IN principle the retinoscopy rack is as well known as the device described above as the three-lens test. Racks are available from $15 \mathrm{in} .-4 \mathrm{ft}$. in length, and from lin.-4in. in breadth. Since the pupil seldom exceeds $6 \mathrm{mms}$. in diameter, and as all text-books are careful to point out, only the central portion of this is considered in retinoscopy, it is unnecessary to employ lenses of a greater diameter than this figure.

Adhering to this principle, it is possible to incorporate in a retinoscopy rack which will fit in the waistcoat pocket the lenses required for the great majority of refractions. Twenty-four lenses ranging from \pm 0.5 to \pm 6.0 in $0.5 \mathrm{D}$ intervals are fitted into an aluminium strip, $16 \mathrm{~cm}$. long, $2 \mathrm{~cm}$. wide, and $0.25 \mathrm{~cm}$. thick. For rapid reading by the light reflected by the retinoscopy mirror, the strength of the lenses is marked in the interspaces, the concave side of the rack coloured red, and the convex black.

In use the rack is extremely rapid, and is so light and small that it causes no unsteadiness when held at arm's length. For cases requiring lenses of a greater strength than $6 \mathrm{D}$. I prefer to use separate lenses, but at the suggestion of J. Benson, of Ediburgh, a cursor can be fitted to extend the range to $\pm 12 \cdot 0$. The rack has 
the failing of all racks, namely that retinoscopy is performed at the distance of half a metre, and that one must be careful to have no other source of illumination behind the user, or the small lenses will be obscured by the reflex. Other users have complained that

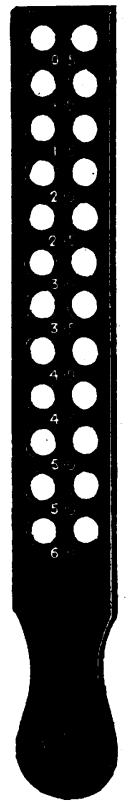

there is too much reflection from the lenses not in use. This difficulty is easily overcome by a slight movement of the hand holding the rack. The rack is so arranged in the latest models that the hand holding it can be rested against the forehead; the weaker lenses are, in consequence, at the end of the rack nearer the hand.

\section{ANNOTATION \\ Surgical Narcosis}

Recently there has been some discussion on the production of what has been termed surgical narcosis for major ophthalmic operations by the administration of drugs which will lessen or remove the distressing psychological effects associated with the operation. It is claimed by some that the mental shock of an ophthalmic operation to elderly persons is the cause of postoperative delirium, flatulence, abdominal pain and absence. of peristalsis. 